\title{
FIBROPLASIA RETROLENTAL
}

\author{
Dr. FERNANDO DONOSO MEDINA \\ Hospital "Roberto del Río".
}

Desde hace algunos años y especialmente en los EE. UU. se ha puesto en evidencia una nueva enfermedad, cuya aparición ha sido posible por la supervivencia que actualmente tienen los niños prematuros nacidos con pesos muy bajos, los que sometidos a rigurosos cuidados pueden alcanzar su total madurez y sobrevivir en buenas condiciones. Se trata de la Fibroplasia retrolental, entidad que hasta el año 1942 habia sido observada esporádicamente, sin distinguirla de otras afecciones oculares. Ese año, Terry ${ }^{1}$, la describe como una enfermedad con caracteres propios.

Basados en los trabajos a nuestro alcance, hemos hecho esta revisión bibliográfica del problema para resumir lo más importante publicado sobre ella hasta este momento.

Todas las definiciones que se han dado de esta enfermedad son más o menos semejantes, así Samilson" dice que es la aparición de una vaina fibrovascular por detrás del cristalino que lleva a la pérdida total de la visión y de causa hasta ahora desconocida; Kinsey y Zacharias ", Ia definen como una enfermedad ocular usualmente asociada a prematuridad, que afecta casi siempre a ambos ojos y se caracteriza por la presencia de una membrana vascularizada situada detrás del cristalino; Terry ${ }^{4}$ agrega a estas definiciones, que la enfermedad se presenta en niños de menos de 3 y media onzas de peso al nacer y que la membrana retrolental es blanca, densa y opaca.

Las cifras de frecuencia relatadas por los diferentes autores varían entre valores que van de $0 \%$ hasta $60 \%$. Así Owens, citado por Samilson ", encontró en un grupo de niños que pesaban al nacer entre 3 y 4 y media onzas, una incidencia del $1,3 \%$; en cambio, Clifford y Weller ${ }^{5}$ dicen que en Boston, donde ellos han trabajado, la enfermedad se presenta en el $23 \%$ de los niños que nacen con pesos entre 907 y $1.361 \mathrm{gr}$. Otros autores mencionan cifras variables; Kinsey y Zacharias dan frecuencias de 10 y $30 \%$; Exline y Harrington ${ }^{1}$ de 10 a $30 \%$ en niños que pesan menos de 1.500 gr. al nacer; Stewart y Clifford, citados por Terry ${ }^{6}$, de $12 \%$ entre los niños que pesaban en el nacimiento $1.360 \mathrm{gr}$. o menos, y Terry ? encontró una incidencia del $12 \%$ en 50 prematuros con pesos de 1.307 gr. o menos al nacer. En resumen, los datos sobre incidencia son variables, presentándose la enfermedad en 1 de cada 40.000 nacidos vivos en USA. ", ocurriendo alrededor de 600 casos al año en la ciudad de Boston", acusando una frecuencia variable en los diversos estudios y que tiene una evidente tendencia al aumento en los EE. UU. ${ }^{3}$.

\section{ETIOLOGÍA}

Es el punto más debatido en esta enfermedad. Se han emitido numerosísimas hipótesis etiológicas y se han publicado frecuentes estudios sobre el origen de ella.

Como esta enfermedad aparece excepcionalmente en niños de término $y$, al contrario, la mayoría de los casos son relatados en niños con pesos muy bajos al nacer, "la prematuridad" tiene una gran importancia en el desarrollo de la enfermedad. Por el hecho de haberse presentado en uno solo de dos gemelos, se ha 
descartado la posibilidad de un factor hereditario en su aparición. Para algunos autores" habría una evidente mayor incidencia de este mal en los niños que presentan hemangiomas cutáneos, existiendo una estrecha relacion entre ambas entidades ", lo cual, sin embargo, no ba sido confirmado.

Existe opinión unánime de que un tipo de lesión semejante a la Fibroplasia retrolental que se presenta en niños de término en el ler. mes de vida y que es predominantemente unilateral, es de origen congénito; en tanto que la lesión de este tipo más común, que es la que se presenta en los prematuros pequeños, es bilateral y no aparece sino hasta después del $3^{\circ}$ o $4^{\text {t }}$ mes de la vida, no es congénita, sino que su desarrollo sería posterior al nacimiento.

También se la ha incriminado a una infección materna por virus en los primeros meses del embarazo sin que exista ninguna evidencia clara de este origen.

Samilson ${ }^{2}$ resume una serie de factores que tendrian importancia en la aparición de esta afección en los niños prematuros:

1. Exposición prematura a la luz: lo que activa los músculos ciliares y pupilares, resultando de ello una falla en la producción o acumulación del humor acuoso, por lo cual el cristalino dependería para su nutrición de la arteria hialoide y de su túnica vasculosa, lo que originaría la persistencia de estas estructuras embrionarias.

2. Carga brusca en el sistema hemovascular del prematuro en el nacimiento: habria en el momento del nacimiento un aumento de la presión sanguínea en el sistema vascular, que junto a la policitemia fisiológica, podrían ser responsables de esta lesión. Reese y Payne creen que la proliferación del tejido vascular tanto en la piel como a nivel del cristalino, serian el resultado de la activación, por falla de la circulación, de restos de tejido angioblástico.

3. Medicación $y$ dieta post-natal del prematuro: la mayoria de las causas a las que actualmente se atribuye un papel etiológico en el desarrollo de la Fibroplasia retrolental, se relacionan con los cuidados inmediatos posteriores al nacimiento de los prematuros pequeños. Es así como Kinsey y Zacharias ${ }^{3}$, Fanconi ${ }^{11}$ y otros, creen que el aumento del uso de vitaminas hidrosolubles, la ferroterapia, el oxígeno a altas concentraciones y otras medidas terapéuticas, tengan un significado etiológico, pero no existen datos suficientes como para extraer una conclusión valedera.

Clifford y Weller "atribuyeron el origen de la afección a una carencia de vitaminas $A$ y D, tanto de la madre como del recién nacido, por lo que administraron en forma profiláctica grandes dosis de estas vitaminas al hijo, sin éxito. Igual cosa les ocurrió a Hepner y col. ${ }^{12}$ quienes además trataron de relacionarla con el uso de sulfamidados en prematuros, sin llegar a conclusiones categóricas.

Otros autores como Laupus y Bousquet ${ }^{14}$ estudiaron la acción de la vitamina $\mathbf{E}$ en la prevención de la Fibroplasia retrolental sin obtener resultados halagadores, ya que la enfermedad se produjo en igual proporción que en el grupo de control, no tratado.

4. Lesiones placentarias: Ingal]s ${ }^{15.1 \mathrm{~B}}$ atribuye importancia etiológica a alteraciones de Ia placenta, a toxemia gravídica y a hemorragias genitales de la madre durante el embarazo, que tendrían como resultado fenómenos de anoxia subletal del feto durante la gestación.

Resumiendo, podemos decir que la etiologia de la Fibroplasia retrolental permanece aún en la obscuridad y que ninguno de los numerosos factores mencionados, con excepción del hecho básico de la prematuridad, puede ser considerado como responsable del origen de esta afección.

\section{ANATOMÍA ParológICA}

La interpretación del cuadro anátomopatológico de esta enfermedad ha sido muy debatido por los diversos autores. Algunos como Blattner ${ }^{s}$ describen un proceso de proliferación de tejido angiomatoso desde la retina que avanza dentro del vítreo y que sería característico del período agudo de la afección. Este período agudo se manifiesta entre la $3^{\text {a }}$ y $5^{\text {a }}$ semana de la vida y está caracterizado por la dilatación de los vasos retinales, aparićión de exudados y hemorragias en la retina, junto con neovascularización de ella y del vítreo. Durante esta etapa podría haber regresión espontánea total de la enfermedad, pero cuando el proceso se encuentra algo más avanzado y aparece tejido fibroso cicatricial, pero vascularizado, que corresponde al segundo período 
anátomo-patológico, las lesiones son irreversibles. Estos mismos autores relacionan estrechamente el hallazgo de este tejido con la presencia de hemangiomas cutáneos que, según ellos, aparecen en otras partes del organismo en el $15 \%$ de los niños con Fibroplasia retrolental, por lo que esta enfermedad sería debida al desarrollo de tejido angiomatoso a nivel del ojo.

Cuando el proceso se encuentra en un estadio más avanzado se manifiesta por la presencia de tejido conjuntivo de tipo embrionario, vascularizado, ubicado por detrás del cristalino, tendiendo los vasos a irradiarse desde un punto central.

La cámara anterior del ojo está muy disminuida o ha desaparecido, siendo además muy frecuente encontrar desprendimientos de la retina $\mathrm{y}$ sinequias, tanto anteriores como posteriores.

\section{SINTOMATOLOGÍA}

Se trata de una enfermedad que se presenta casi con exclusividad en niños prematuros $y$ Hepner y col. ${ }^{12}$ en una serie de 66 casos, encontraron que el 99\% eran prematuros y gran parte de ellos lo eran de muy bajo peso de nacimiento.

A pesar de no haberse descrito predilección por sexo, Exline $\mathrm{y}$ Harrington * encuentran sólo un $38 \%$ de varones $y$ destacan el hecho de que en la muestra analizada, el $83 \%$ de los niños eran de raza negra.

La enfermedad comienza a manifestarse, en general, entre el $1^{\circ}$ y el $5^{\circ}$ mes de la vida, pero en una serie de 56 prematuros, Jefferson ${ }^{1 s}$ encuentra un caso en que la aparición de las lesiones fué a los 8 dias de edad.

La lesión es bilateral entre el 12 y $18 \%$ de los casos según Samilson ${ }^{2}$.

Los signos de esta enfermedad deben ser buscados ya que de otra forma las lesiones suelen pasar desapercibidas. Lo primero que aparece es un hundimiento de los párpados que se encuentra en relación con la microltalmia que casi siempre se encuentra presente. El iris se aprecia de un color sui generis denominado por Terry "azul fetal", que puede tener ligeras variaciones hacia el azul gris o al café sucio. A veces puede verse un nistagmo completo, con un movimiento rotatorio lateral de la cabeza. Puede exjstir además fotofobia y en estos casos los niños se cubren los ojos con sus manos cuando son expuestos a la luz.
Al examen oftalmoscópico lo primero que se manifiesta es un llenamiento exagerado de las venas retinales acompañado de aumento de la tortuosidad de las arterias, lo que es seguido del mismo fenómeno en los vasos venosos, que se alteran en tal forma, que pueden llegar a formar verdaderos arcos o aún ovillos. Estos procesos van ocurriendo en forma parcelar, de tal modo que las alteraciones de la retina se van produciendo primero en un segmento y luego en los siguientes, pasando varjos días antes que la lesión comprometa un segmento vecino. Pronto la retina se pone edematosa y comienzan a aparecer vasos sanguineos de neoformación, especialmente en la periferia de ésta, dando paso a la formación de un tejido opaco, grisáceo y vascularizado, por detrás de los cristalinos, donde las bandas parecen irradiarse desde un punto central.

La cámara anterior del ojo es poco profunda o se halla borrada. Los procesos ciliares scn delgados y se aprecian por delante del tejido opaco, cuando son visualizados desde el ángulo periférico.

Magnusson ${ }^{1} i$ encontró junto a la lesión oftálmica algunos casos con evidente retardo en el desarrollo mental y mediante la ventriculografía pudo comprobar la existencia concomitante de lesiones cerebrales. Lo mismo afirma Krause, citado por Samilson", pero este mismo autor se encarga de desmentir tales afirmaciones al aseverar que en la gran mayoría de los casos esto no sucede y que el retardo mental de algunos de estos prematuros se debería a la calidad de la enseñanza que se les proporciona. Terry ' quien encuen. tra en 3 casos de Fibroplasia retrolental un discreto retardo mental, piensa que sería sólo una coincidencia, ya que este hecho esta presente en el $10 \%$ de todos los niños prematuros.

La evolución que sigue esta enfermedad es progresiva y la regresión ha sido descrita en pocos casos y sólo en niños que acusaban una enfermedad en comienzo. Cuando ya aparecen lesiones de tipo cicatricial ninguna posibilidad de regresión espontánea existe. Como esto ocurre en la mayoría de los niños y siendo la afección muchas veces bilateral (12 a $18 \%$ ), la pérdida total de la visión es su consecuencia, afirmando algunos autores que el $33 \%$ de los niños ciegos de edad pre-escolar en los EE. UU, deben su ceguera a la Fibraplasia retrolental. 


\section{DIAGNósTico}

En cuanto al diagnóstico de este cuadro, existen dos aspectos que para Hepner y col. ${ }^{12}$ tienen bastante importancia; se trata, por una parte, de la bistoria clínica en que los datos de existir: a) prematuridad, bj ceguera ccurrida entre el $1^{\circ}$ y $6^{\circ}$ mes de la vida y c) antecedentes de ceguera o enfermedades oculares similares en los familiares, pueden orientar al clínico hacia este diagnóstico. Por otra parte, el examen físico completo del ojo, confirma la sospecha clínica. Sin embargo, Blattner " insiste en que el diagnóstico clínico es dificil y que sólo sería el examen oftalmoscópico, efectuado por un especialista competente, lo que corroborará tal hipótesis.

Entre los principales diagnósticos diferenciales que pueden plantearse, ante un enfermo de esta naturaleza, tenemos el Retinoblastoma, eI Desprendimiento seroso de la retina y la Catarata congénita. En el Retinoblastoma nunca el cristalino se halla tomado desde un comienzo, el ojo tiene un color amarillento y no se encuentra alterado en su tamaño. En el Desprendimiento seroso de la retina, la lesión no es del cristalino y además el ojo es fácilmente transiluminado. En cuanto a la Catarata congénita, se observa al examen una opalescencia lechosa del cristalino, en un ojo de tamaño normal y en algunos casos se puede obtener en estos enfermos el antecedente de rubeola de la madre en el primer trimestre del embarazo.

\section{TRATAMIENTO}

Son múltiples las terapias ensayadas en esta enfermedad, pero a decir verdad, ninguna de ellas ha logrado un éxito demostrado y satisfactorio.

Owens y Owens propusieron la administración de grandes dosis de vitamina $\mathrm{E}$ ( $150 \mathrm{mgr}$. por día), pero no lograron éxito después de un período de ensayos de 1 $y$ medio años.

La cortisona y el ACTH, que parecieron dar resultados halagadores en un comienzo, también fracasaron posteriormente l:-s:

Hepner y col. ${ }^{21}$ ensayaron el uso de la leche humana como prevención de la enfermedad, pero la afección se presentó en igual proporción que en el grupo de control alimentado con leche de vaca.

Blattner ${ }^{8}$, que utilizó como terapia para esta enfermedad la extirpación quirúr- gica de las membranas opacas, tuvo un resultado desalentador ya que también existe en estos casos la degeneración de la retina, por lo cual la visión no puede ser restituída por ningún medio.

Además de los tratamientos mencionados, se ha ensayado la radioterapia, la diatermia, la escisión de las membranas, la remoción del cristalino, la incisión de los cuerpos ciliares, etc., logrando todos estos medios sólo un éxito discreto.

En vista del fracaso de la mayoría de las terapias empleadas, Terry " insiste en la prevención de las complicaciones, especialmente el glaucoma secundario y las sinequias, mediante el empleo de substancias adecuadas. Entre las medidas tendientes a prevenir la aparición de la enfermedad, aconseja mantener a los niños prematuros de bajo peso de nacimiento en una pieza cbscura o alumbrada con luz roja y evitar el exceso de transfusiones, vitaminoterapia, ferroterapia y la oxigenoterapia, a alta concentración y por tiempo prolongado.

\section{BIBLIOGRAFÍA}

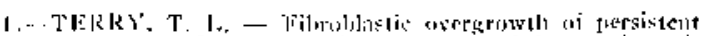

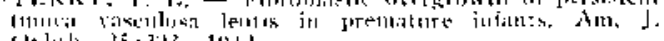
(J)lith. $25: 3: 13,1^{13}+2$.

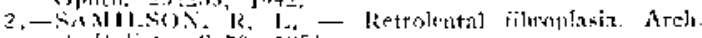

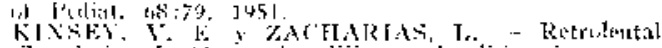

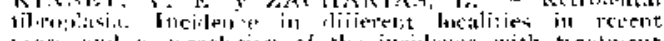

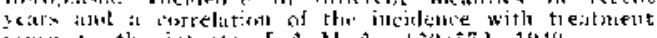

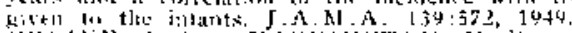

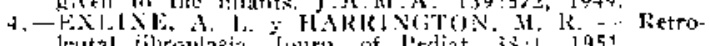

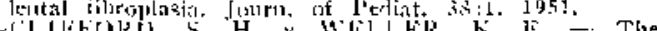
abrsejution of vitanin a iu prematurely horit infants.

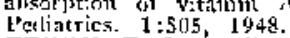

r. 一TFKRY, T. I. - Retroiental fibroplasia. IV. Cisneral comsiderations, J.A.M. A. 128:532, 19+5.

7.-TEKRS, T. I, - Ketrolental filoroplasia in prematute infants. Arch. Oplat?. 3.3:3i)?, 194.

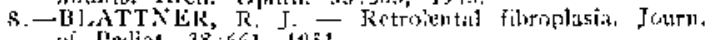

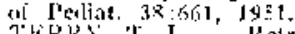

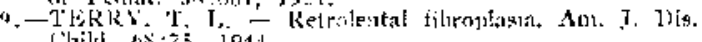
chilt, bis ist.

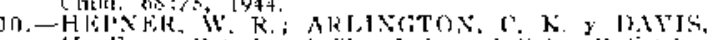

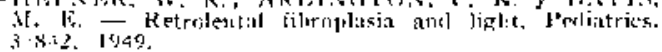

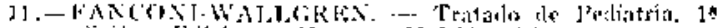

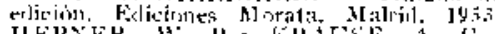

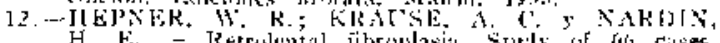

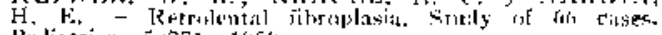

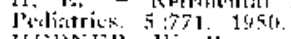

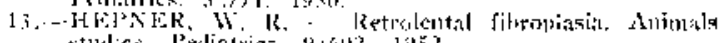
stmlice. J'ellialrics. $9: 41 \% 2,1052$.

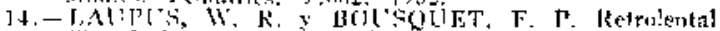

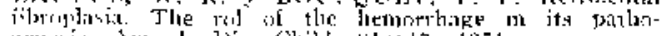

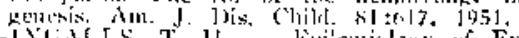

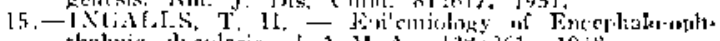

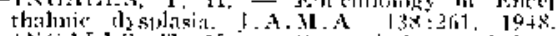

16. - INGASAS, F

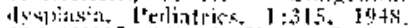

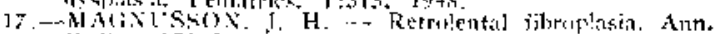

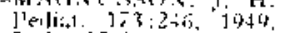

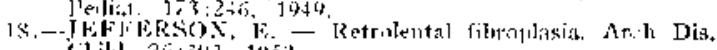
(1.ili. $240: 312,1452$.

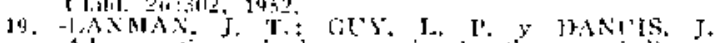

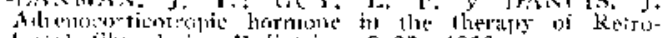

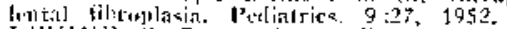

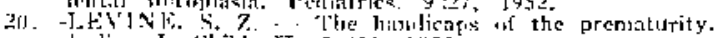

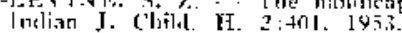

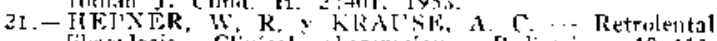

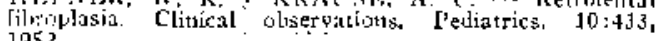
1952. 